\title{
E- Farming: A Breakthrough for Farmers
}

\author{
Samer D.M, Subramaniya Raman M.K \\ SVCE, Sriperumbudur, \\ India -602117
}

\begin{abstract}
Today, mobile phones are used everywhere, and android is the primary operating system dominating the mobile operating system market field with a market share of more than $80 \%$ and most of the applications are free to download. We are targeting the horticulture sector in which smartphones can be used to provide the farmer with the details of all the different types of crops that he can harvest and also the best efficient way in which he can get the yield. All this information will be provided in regional audio form also so that it will be easy for farmers to understand. For example, it can be extremely useful for the farmers in India as he/she will get information in multiple languages within a few key presses. Even an illiterate person can use this app easily.
\end{abstract}

\section{INTRODUCTION}

Some problems are identified after completing incentive study of this topic:

1. Various website provides Horticulture information related to fruit, flowers, and vegetables. But all these data are in English Language and in Text form. [8] That is why Indian farmers who are illiterate are not able to take advantage of these services. Even If that person wants to, he needs a laptop or other device which makes him/her dependent on someone.

2. IFFCO Kisan Sanchar Limited (IKSL) and Routers Marker Light (RML) are the agencies that are currently providing agriculture information via SMS and Call. But they take money for the usage of their services and don't work where mobile network is not available. This application also gives additional features like Government Notifications, Bank notifications etc.

3.Nidhi Dwivedy has proposed that:

Decision Support Systems are more important and usually avoids risk developing environments. It has been suggested that the WTO is stipulating reductions for export subsidies on farm products and will make Indian exports more competitive. It has been estimated that the export potential may increase up to $\$ 1.5$ billion by 2020 . The advantage of the emerging order, is that the Indian farmer needs to be equipped with information that have been facilitated by undertaking a proper[2] SWOT analysis and its comparison may led to conventional wisdom and satisfy himself on an appropriate course of action. The Available information does not satisfy which projects on the weaknesses of the adverse effect of WTO on any specific agricultural product will help in taking the necessary corrective measures. In the present scenario, the competitive advantage is necessarily required to be fully exploited for increasing the export potential.

\section{Market and price information}

The following are examples of initiatives to improve food production and security by improving market linkages and access to local and international markets for smallholder farmers' products. They rely on several levels of information generation and dissemination, including at the farmer and community levels:

Rural Knowledge Network Pilot Project for East Africa: This project encompasses market access networks in Kenya, Tanzania and Uganda, with actors at national, district and local levels who keep a constant and effective communication link (e.g. via e-mail, telephone, SMS, faceto-face meetings, Internet) for information-sharing and business-to-business learning. Initiated by FAO, the pilot project ran from 2007 to 2010; its most well-established and active offspring is Agri Net Uganda.38 Market Linkages Initiative Bridging Activity (Malawi):[1][5] This USAIDfunded project helped improve post-harvest handling, developed a transparent trading environment and increased access to accurate market information for smallholder farmers through a partnership with Esoko Networks and the Agricultural Commodity Exchange. Women groundnut producer farmers were trained in ICT skills for sourcing market information via an Esoko SMS-based subscription service, and consequently were able to sell their produce at a fairer price, organize transport in more cost-effective ways and quintuple their earnings.39 Krishi FM (Nepal): A rural radio relies on farmer-generated market information to inform its listeners. Farmers who roam or sell at local markets phone in to the local community radio to share information on current market prices. SIM-Agri (Burkina Faso): IICD and its partners launched SIM-Agri, a platform giving 3000 farmers access to vital market information via mobile phones and computers. Market price collectors around the country gather information at various farmers markets and then send information about prices and products at those markets to the SIM-Agri platform.[9] For the price of a regular text message, the farmers can ascertain the prices of their crops in various markets and learn when and where they can earn the best price for their produce.

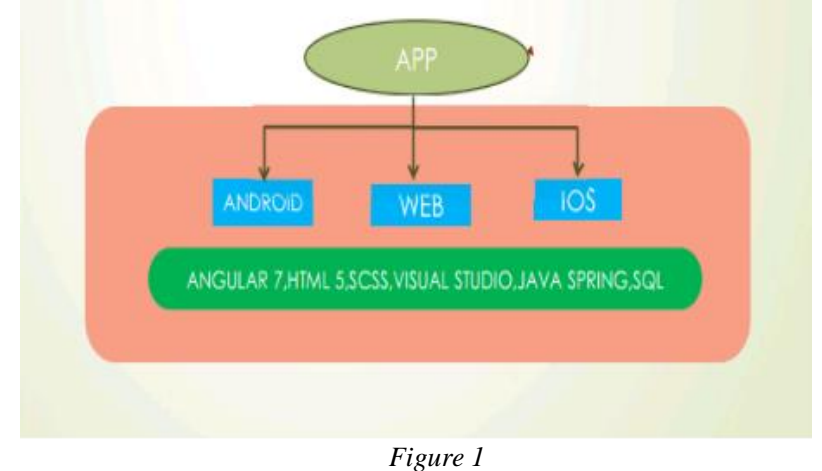




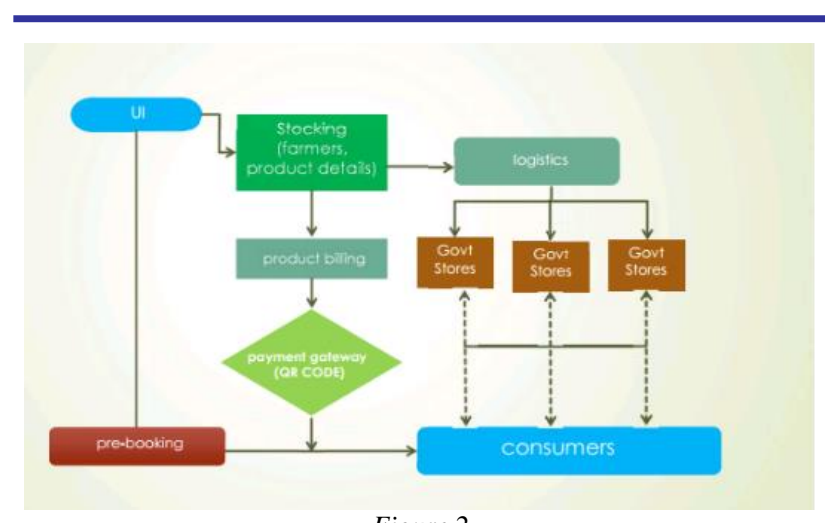

Figure 2

\section{Stage wise Implementation:}

Our project was divided into different stages or can say modules, these modules helped to decide the actual flow of the application and implementation of the project was achieved smoothly with the modular approach.[5][7] The modular Approach was achieved with the help of the class diagram scheme.

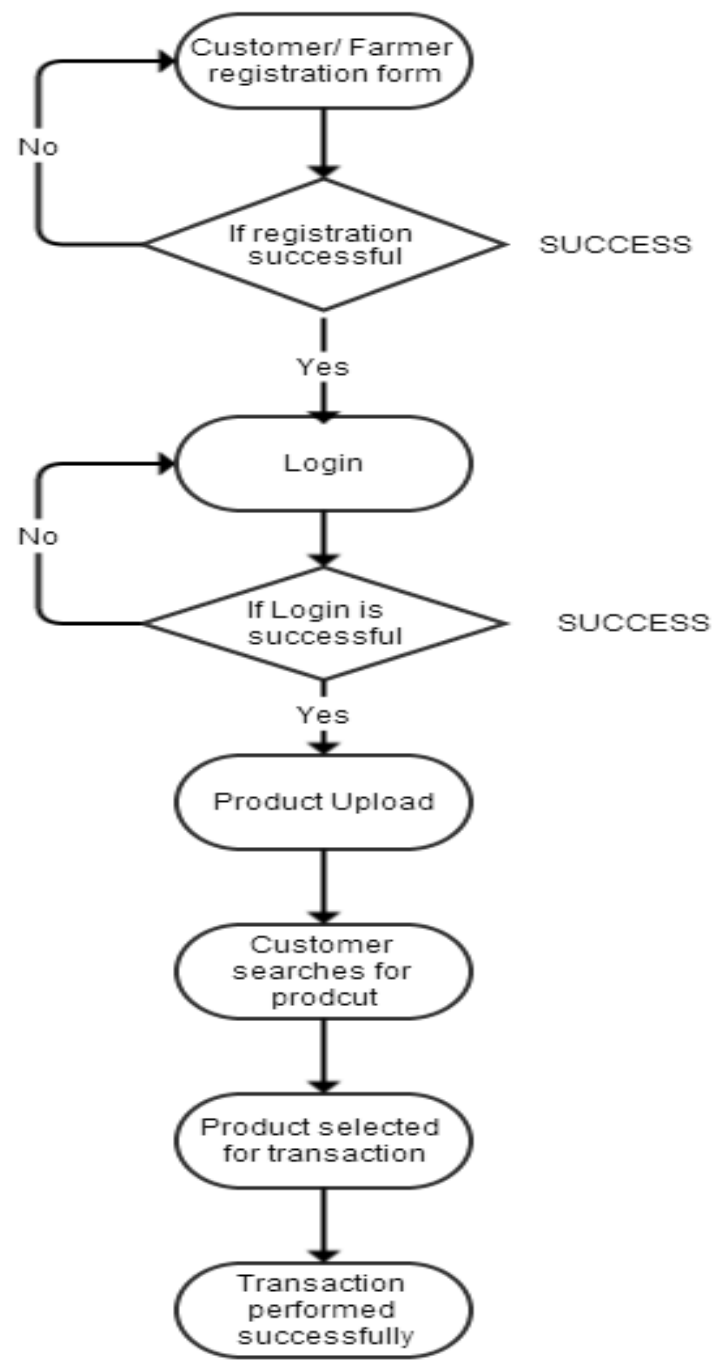

Figure 3

\section{Implementation Stage:}

After the development of the individual modules of the project we looked in to the implementation of each of the module and defined the relation between them, this was achieved by the use of flow charts which described the functions of each of the modules used in the project.[3][4]

\section{Cloud Implementation:}

Working of the Google App Engine. The implementation scheme of the project was categorized as per the configuration of the google app engine.

\section{Customer Registration:}

The Customer Registration process involves that the customer enter his personal details and one or more required fields those are then validated by the application and the success or failure of registration is decided if the registration process fails then the customer has to register again of course with the genuine details required for the registration process.

\section{Farmer Registration:}

The Farmer registration process is similar to the Customer registration process the customer also has to fill genuine details in order to successfully perform the transaction process of the farming process.

\section{E-Farming Process Implementation:}

This is the most important phase of the project development this phase provides the implementation of the farming process it provides details on how the farming process will be carried out efficiently.

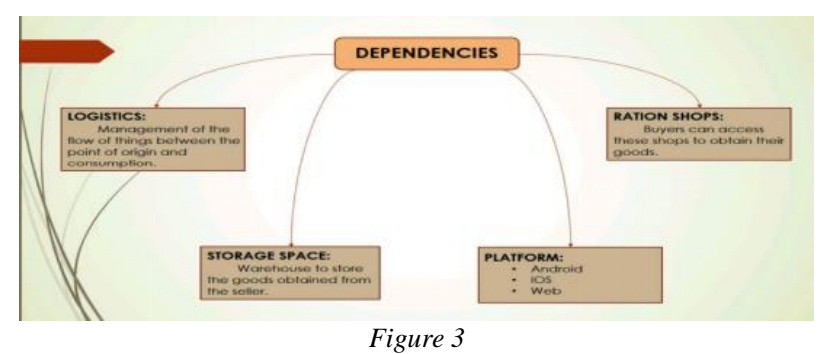

Angular platform:

Angular has proved to be a magnificent framework in recent times. Its simplicity, developer-friendliness, and ability to build awesome projects have attracted many freshers as well as experienced developers to learn Angular.

Angular is a cross platform language. It supports multiple platforms. You can build different types of apps by using Angular.

1.: Angular facilitates you to create desktop installed apps on different types of operating systems i.e. Windows, Mac or Linux by using the same Angular methods which we use for creating web and native apps.

2. You can build native apps by using Angular with strategies from Cordova, Ionic, or Native Script.

3. Progressive web applications are the most common apps which are built with Angular. Angular provides modern web platform capabilities to deliver high performance, offline, and zero-step installation apps. 


\section{Efficient Performance:}

Angular is amazingly fast and provides a great performance due to the following reasons:

1.Universal support: Angular can be used as a front-end web development tool for the programming languages like Node.js, .Net, PHP, Java Struts and Spring and other servers for near-instant rendering in just HTML and CSS. It also optimizes the website for better SEO.[9][3]

2.Code splitting: Angular apps are fast and loads quickly with the new Component Router, which delivers automatic code-splitting so users only load code required to render the view they request.

3.Code generation: Angular makes your templates in highly optimized code for today's JavaScript virtual machines which gives the benefits of hand-written code.

\section{Visual Studio:}

Microsoft Visual Studio is an integrated development environment (IDE) from Microsoft. It is used to develop computer programs, as well as websites, web apps, web services and mobile apps. Visual Studio uses Microsoft software development platforms such as Windows API, Windows Forms, Windows Presentation Foundation, Windows Store and Microsoft Silverlight. It can produce both native code and managed code.

Visual Studio includes a code editor supporting IntelliSense (the code

completion component) as well as code refactoring. The integrated debugger works both as a source-level debugger and a machine-level debugger. Other built-in tools include a code profiler, forms designer for building GUI applications, web designer, class designer, and database schema designer. It accepts plug-ins that enhance the functionality at almost every level-including adding support for source control systems (like Subversion and Git) and adding new toolsets like editors and visual designers for domain-specific languages or toolsets for other aspects of the software development lifecycle (like the Team Foundation Server client: Team Explorer).

SQL: Structured Query Language) is a domain-specific language used in programming and designed for managing data held in a relational database management system (RDBMS), or for stream processing in a relational data stream management system (RDSMS). It is particularly useful in handling structured data where there are relations between different entities/variables of the data. SQL offers two main advantages over older $\mathrm{read} /$ write APIs like ISAM or VSAM. First, it introduced the concept of accessing many records with one single command; and second, it eliminates the need to specify how to reach a record, e.g. with or without an index. Cascading Style Sheets (CSS) is a style sheet language used for describing the presentation of a document written in a markup language like HTML. CSS is a cornerstone technology of the World Wide Web, alongside HTML and JavaScript.CSS is designed to enable the separation of presentation and content, including layout, colors, and fonts. This separation can improve content accessibility, provide more flexibility and control in the specification of presentation characteristics, enable multiple web pages to share formatting by specifying the relevant CSS in a separate .css file, and reduce complexity and repetition in the structural content.

\section{CURRENT AND FUTURE CHALLENGES}

While substantial progress has been made in making ICTs available and accessible for rural communities, challenges remain in respect to the following eight critical factors for success:

1. Content: Adaptation of content to local needs, languages and contexts remains challenging. Appropriate information resources (i.e. content) and trusted intermediaries are necessary for the success of e-agriculture initiatives. Dissemination of information may be constrained if the nature of information does not match farmers' needs in terms of format and relevance.[5] While ICTs can deliver large amounts of information, this does not imply effective use of it. Locally adapted content and existing relationships based on trust are not yet given sufficient attention and priority in development plans. Bringing ICTs and development planning closer together, with information innovations coming directly from the rural communities themselves, remains an often-overlooked design consideration in meeting the demands of the poorest communities.

2. Capacity development. This is comprised of three dimensions: the enabling environment, the organizational capacity and individual's capacity. Capacities at the individual, organizational and institutional levels need to be strengthened.[7] The focus on improving access to agricultural information without addressing the ability to effectively use the information has not yet yielded the desired reduction of the rural digital divide. Illiteracy, limited skills in using complex devices to search for information and cultural issues remain barriers to effectively receiving and using information delivered via ICTs. Models of capacity development need to be based on social characteristics, information needs and the function of technology in context. Scaling up pilot ICT projects to reach millions of smallholder farmers remains a challenge. Upscaling and mainstreaming of projects is often not sufficiently supported by dialogues at organizational and national levels which could create a policy environment conducive to the effective use of ICTs in agriculture. The price of access to ICTs can be extremely high in some countries.[5] Pricing of broadband or mobile services is an important barrier for most vulnerable groups, such as women, youth, older farmers, and people living in most remote areas Gender and diversity. Access and opportunities are not distributed equitably among users, creating asymmetries that must be addressed with specific policies targeting the source of the inequalities. Access for women, youth, older farmers, and people living in the most remote areas is hindered by the price of access to ICTs (e.g. broadband or mobile services) and by persistent inequalities. Gender inequalities remain a serious issue in the digital economy, as does the gap between urban and rural populations. The digital divide is not only concerned with technological infrastructure and connectivity; it is a 
multifaceted problem of ineffective knowledge exchange and management of information content; insufficient human resources and institutional capacity; and lack of sensitivity to gender and the diverse needs of different groups.[8][5] For example, illiterate and older farmers often have lessdeveloped digital skills, and so they are usually less likely to adopt ICTs.Many of the factors that constrain male farmers in adopting more sustainable and productive practices restrict women even more. Specific gender barriers further limit women farmers' capacity to innovate and become more productive. Youth's access and familiarity with technologies, as well as their role in the social dynamics of rural communities, are not yet sufficiently leveraged.

4. Access and participation. Access to ICTs is not yet equitable. As mentioned before, a gender-based digital divide persists, and is more frequent in rural than urban areas.[3][2] The digital divide between men and women is increasing, despite the growing number of Internet users. Improved access to ICTs alone will not resolve the gender digital divide. As with the challenges reported in other key areas, proper design and implementation based on a bottomup and participatory approach that involves the communities themselves can reduce the potential for information inequity that can be created when introducing new ICTs into a community.

5. Partnerships. Public-private partnerships are recognized as a critical factor in sustainable business models at the community level, but these do not always have to be with large corporate firms; small, local private companies, local producer organizations and community-based NGOs often have the social capital to provide trusted information and good quality services. Diverse advisory and extension services offered by different types of providers are more likely to meet the various needs of farmers, as there is no one type of advisory service that can fit all circumstances.[4] With a broader variety of potential partners comes a new challenge: the formal recognition of information and service quality standards, and the partners' agreement to be held accountable for meeting them.

6. Technologies. Identifying the right mix of technologies that are suitable to local needs and contexts is often a challenge, despite - or because of - the rapid increase in mobile telephone penetration in rural areas. While this offers great potential for increasing access to information, challenges remain in the area of effective use of mobile telephony that are related to access and capacity as described above Technologies should be suited to local contexts and needs, and their selection should increasingly take into account the influence ICTs have on gender and social dynamics. The appropriation of ICTs by youth in support of farming activities is also creating shifts in the social dynamics between youth and older community members, or between rural and urban/peri-urban communities.[11]

7. Economic, social, and environmental sustainability. Scaling up pilot ICT projects to reach millions of smallholder farmers and identifying sustainable business models are still challenges. On the one hand, pricing is critical to sustainable agribusiness models at the community level. Investments are needed to cover the cost of creating content and collecting data. On the other hand, social sustainability can be hindered if clear roles and responsibilities have not been clarified among stakeholders. For example, the location of an ICT center should be socially convenient for all users (including women and older people). Finally, technology waste is an issue and a polluting factor that should not be underestimated.[15] Measurements and data on the impact of mobile technologies on agriculture are scant and generally anecdotal. Solid information is needed regarding the impact of previous initiatives, including lessons learned, to inform the design and approach of future efforts. At the same time, these impacts are inherently difficult to measure because they may not be immediate or may not be reported or recorded.[16] Often, success of ICT interventions in agriculture is on a case-by-case basis. Sustainability of ICTs for agriculture initiatives may be at risk if development organizations, governments and the private sector fail in defining indicators and data that validate investments in ICTs and the positive results these may have.

\section{RESULTS}

The occupational structure of India is dominated by the "agricultural sector" and the "manufacturing sector" and the "service sector" is lagging far behind in this context. This shows that India is predominantly an agricultural economy and hence it requires strongest protection and development of its "agricultural resources". India is facing certain "Agricultural Challenges" that must be resolved as soon as possible. The major challenges to "Agriculture Sector in India" are: (a) Insufficient agricultural infrastructure and support facilities, (b) Insufficient institutional capacity to deliver farmers specific services, (c) Lack of awareness regarding suitable agricultural methods among the farmers, (d) Agricultural content development and its upgradations, (e) Ownership issues of the public and government generated data, (g) Inadequate use of Public-Private Partnerships in India, (h) Lack of "Common Platforms" for the farmers in India, (i) Absence of an "Agricultural ThinkTank" in India, (j) Insufficient use of ICT for agricultural purposes, etc. The Government of India must come up with "Suitable Policies" and "Incentives" for the farmers so that they may be motivated and encouraged to give their best. This must be supported by the use of ICT that can-do wonders for the agriculture sector of India. ICT can be used to improve the lives of the rural communities by leveraging of agriculture outputs through technological interventions. Some of the benefits of ICT for the improvement and strengthening of agriculture sector in India are: (a) Timely information on weather forecasts and calamities, (b) Better and spontaneous agricultural practices, (c) Better marketing exposure and pricing, (d) Reduction of agricultural risks and enhanced incomes, (e) Better awareness and information, (f) Improved networking and communication, (g) Facility of online trading and e-commerce, 3 (h) Better representation at various forums, authorities and platform, etc. There is a "Digital Divide" that is hindering the capacity and productivity of rural agricultural activities carried out by the marginalized farmers in India. The situation of marginalized farmers of rural India can improve if we pay attention 
towards the following capacity development initiatives: (a) Conducive Legal Framework, (b) Simple and Farmer Friendly Governmental Regulatory Measures, (c) Governance and Institutional Reforms, (d) Transparency in various dealing, (e) Fixing of Accountability of Persons/Institutions dealing with Marginalized Farmers, (f) Representation of Marginalized Farmers in various matters affecting them, (h) Policy and Strategy Reforms in India, (i) Providing of Technical Assistance to Rural Credit Cooperative Banks (RCCBs), (j) Use of ICT for Banking and Financing Purposes, (k) Improved Managerial and Operational Capacities, (l) Self-Regulation and SelfManagement of Indigenous Issues, (m) Affordable Financial Support, (n) Banking Solutions for Poor Rural Farmers through Franchisee Agreements with micro-credit institutions must be made, etc. India, like others, is facing a rising demand for food grains that may not be fully met by the supply side.

\section{CONCLUSION}

Based on the results obtained from the above, the following conclusions were made:

- Majority of farmers in the state or country are not aware that mobile phones can be used to conduct businesses and receive information.

- Mobile phone costs should be lowered to enable majority of farmers for having access to the current information about the agriculture business within the state or country.

- e-Agriculture has not been implemented because farmers in the country have not been sensitized about it and young farmers were in lack of information about the agriculture such that eagriculture might provide them useful information's regarding the plantations that they have grown.

- The government should also conduct workshops/seminars to create awareness for the farmers on how to avail these benefits.

\section{REFERENCES}

[1] Peter Namisiko and Moses Aballo "Current Status of e-Agriculture and Global Trends:A Survey Conducted in TransNzoia County, Kenya" in International Journal of Science and Research Volume 2 Issue 7, 2013

[2] Marcel Fafchamps and Bart Minten "Impact of SMS-Based Agricultural Information on Indian Farmers" in Oxford journals VOL. 26, NO. 3, pp. 383-414, 2012

[3] Lobo, S., Doke, P., Kimbahune, S. (2010, October). GappaGoshti: a social networking platform for information dissemination in the rural world. In Proceedings of the 6th Nordic Conference on Human- Computer Interaction: Extending Boundaries (pp. $727-$ 730). ACM. 30

[4] Ramamritham, Krithi, Anil Bahuman, Ruchi Kumar, Aditya Chand, Subhasri Duttagupta, GV Raja Kumar, and Chaitra Rao. "aAQUA-A Multilingual, Multimedia Forum for the community." In IEEE International Conference on Multimedia and Expo, vol. 3 2004.

[5] D. W. Allen and M. Anderson Ochs. "Building Pathways out of Rural Poverty through Investments in Agricultural Information Systems.

[6] Ginige, T., \& Richards, D. A model for enhancing empowerment in farmers using mobile based information system.

[7] Smitha K K and Chitharanjan K. Article: Security of Data in Cloud based E-Governance System. IJCA Special Issue On Advanced Computing and Communication Technologies for HPC Applications ACCTHPCA (2):1-6, July 2012. Published by Foundation of Computer Science, New York, USA

[8] Hongdong Guo. - An analysis about factors which affect farmers.

[9] LUAN Jingdong and CHENG Jie;Establishment of the Agricultural Risk Management System Based on Industrial Chain[J]; Issues in Agricultural Economy;2007-03

[10] Google App Engine API, https://appengine.google.com/ [11] Google App
http://www.en.wikipedia.org/wiki/Google_App_Engine Engine

[12] Fielding, R. Architectural Styles and the Design of Network-based Software Architectures, University of California, 2000.

[13] Alarcon, R., Wilde, E., \& Bellido, J. Hypermedia-driven RESTful Service Composition, Published in the ICSOC 2010 International Workshops PAASC, WESOA, SEE, and SC-LOG San Francisco, CA, USA

[14] Erlang Programming Language, http://www.erlang.org/

[15] Lomotey, R. K., Yiding Chai, Kazi Ashik Ahmed, and Deters, R. 2013. Distributed Mobile Application for Crop Farmers. Proc. of the International Conference on Management of Emergent Digital EcoSystems (MEDES 13), pp:5 pages, Neumünster Abbey, Luxembourg, October 28th till October 31th, 2013

[16] Lomotey, R. K., Yiding Chai, Kazi Ashik Ahmed, and Deters, R.2013. Web Services Mobile Application for Geographically Dispersed Crop Farmers. Proc. of the IEEE 16th International Conference on Computational Science and Engineering (CSE 2013), pp:8 pages, Sydney, Australia Dec. 3-5, 2013 